\title{
VARIATION OF MULTIPARAMETER BROWNIAN MOTION
}

\section{LANE YODER}

ABSTRACT. Lévy's $N$-parameter Brownian motion in $d$-dimensional space is denoted by $W^{(N, d)}$. Using uniform partitions and a Vitali-type variation, Berman recently extended to $W^{(N, 1)}$ a classical result of Lévy concerning the relation between $W^{(1,1)}$ and 2-variation. With this variation $W^{(N, d)}$ has variation dimension $2 N$ with probability one. An appropriate definition of weak variation is given using powers of the diameters of the images of sets which satisfy a parameter of regularity. A previous result concerning the Hausdorff dimensions of the graph and image is used to show the weak variation dimension of $W^{(N, d)}$ is $2 N$ with probability one, extending the result for $W^{(1,1)}$ of Goffman and Loughlin. If unrestricted partitions of the domain are used, the weak variation dimension of a function turns out to be the same as the Hausdorff dimension of the image.

In a recent paper by Goffman and Loughlin [3] strong and weak variations were defined, and the strong and weak variation dimensions of Brownian motion were shown to be 2 with probability one. The definitions and proofs given in that paper do not immediately generalize to multiparameter Brownian motion.

The purpose of this paper is to find appropriate definitions of strong and weak variations and, using a previous result on the Hausdorff dimensions of Brownian motion (see the appendix for a summary of the proof), to show for $N$-parameter Brownian motion in $d$-dimensional space, both the strong and weak variation dimensions are $2 N$ with probability one.

The distinction between the strong and weak variations becomes more pronounced in higher dimensions. In $N$ parameters the strong variation is defined in terms of the vertices of rectangles and the weak variation in terms of the oscillation on the rectangle, whereas in the one variable case both the strong and weak variations may be defined in terms of the oscillation on an interval.

Presented to the Society May 3, 1973; received by the editors July 27, 1973. AMS (MOS) subject classifications (1970). Primary 60G17. 
Let $W^{(N, d)}$ denote Lévy's $N$-parameter Brownian motion with values in $d$-dimensional Euclidean space; i.e., if $X=W^{(N, d)}$, then $X(t, \omega)=$ $\left(X_{1}(t, \omega), \ldots, X_{d}(t, \omega)\right) \in \mathbf{R}^{d}$, where $t=\left(t_{1}, \ldots, t_{N}\right) \in \mathbf{R}^{N}$ and the coordinate functions $X_{i}$ are mutually independent, separable Gaussian processes with mean zero and covariance

$$
E\left(X_{i}(s), X_{i}(t)\right)=1 / 2[|s|+|t|-|s-t|] .
$$

Here $|\cdot|$ is the Euclidean norm.

1. Let $a=\left(a_{1}, \ldots, a_{N}\right)$ and $b=\left(b_{1}, \ldots, b_{N}\right)$ be vertices of a rectangle in $\mathbf{R}^{N}$, and let $Y$ denote the $N$ th-order difference of the sample function $X=W^{(N, d)}$ over this rectangle:

$$
Y=\Delta_{1} \cdots \Delta_{N} X=X(a)-\sum_{r=1}^{N} p_{r}+\sum_{r<s} p_{r s}-\cdots+(-1)^{N} X(b)
$$

where $p_{r s} \ldots t$ denotes $X\left(c_{1}, \ldots, c_{N}\right)$ with $c_{r}, \ldots, c_{t}$ equal to $b_{r}, \ldots, b_{t}$, respectively, and the remaining $c_{j}$ equal to $a_{j}$. In other words $Y$ is the sum of the function $X$ taken at the vertices of the rectangle, where $X$ has opposite signs for every pair of vertices sharing a common edge.

Let $\pi=\left\{A_{i}\right\}$ be a partition of the unit $N$-cube, $U^{N}$, into rectangles $A_{i}$. We define the strong $p$-variation of $X$ to be

$$
V_{p}^{s}(X)=\sup _{\pi} \sum_{i}\left|Y_{i}\right|^{p}
$$

where $Y_{i}=\Delta_{1} \cdots \Delta_{\dot{N}} X$ defined for the rectangle $A_{i},|\cdot|$ is the Euclidean norm in $\mathbf{R}^{d}$, and the sup is taken over all partitions of $U^{N}$ into rectangles.

Then either $V_{p}^{s}(X)=\infty$ for all $p \geq 1, V_{p}^{s}(X)<\infty$ for all $p>1$, or there is a unique $p>1$ such that $V_{q}^{s}(X)<\infty$ for all $q>p$ and $V_{q}^{s}(X)=\infty$ for all $q<p$. This gives the strong variation dimension of $X, \operatorname{dim}_{s}(X)$, which is $\infty, 1$, and $p$, respectively, in the three cases.

Theorem 1. For $X=W^{(N, d)}, \operatorname{dim}_{s}(X)=2 N$ with probability one.

Proof. This follows immediately from two known properties of $W^{(N, d)}$.

(a) For almost every $\omega, X(\omega)$ is in the Lipschitz $\delta$ class for every $\delta<1 / 2$ [5]. Let $p>2 N$ and $\delta=N / p$. Let $\pi_{k}=\left\{A_{i}^{k}\right\}$ be any sequence of partitions of $U^{N}$ into rectangles and let $l_{i}^{k}$ be the length of the shortest edge of $A_{i}^{k}$. Then the $N_{\text {th }}$ order difference $Y_{i}^{k}$ defined for $A_{i}^{k}$ satisfies $\left|Y_{i}^{k}\right| \leq 2^{N-1} K\left(l_{i}^{k}\right)^{\delta}$ and

$$
V_{p}^{s}(X) \leq \limsup _{k \rightarrow \infty} \sum_{i}\left|Y_{i}^{k}\right|^{p} \leq\left(2^{N-1} K\right)^{p} \underset{k \rightarrow \infty}{\lim \sup } \sum_{i}\left(l_{i}^{k}\right)^{N}
$$


The sum $\Sigma_{i}\left(l_{i}^{k}\right)^{N}$ is bounded since the Hausdorff $N$-measure of $U^{N}$ is finite. Thus $\operatorname{dim}_{s}(X) \leq 2 N$ with probability one.

(b) Let $\pi_{k}=\left\{A_{i}^{k}\right\}$ be the partition of $U^{N}$ into cubes of edge length $2^{-k}$. Berman generalized a classical result of Lévy by showing that there is a constant $B_{N}>0$ such that for $X=W^{(N, 1)}$

$$
\lim _{k \rightarrow \infty} \sum_{i}\left|Y_{i}^{k}\right|^{2 N}=B_{N}
$$

with probability one [1]. Thus for $X=W^{(N, d)}, \operatorname{dim}_{s}(X) \geq 2 N$ with probability one.

2. The strong $p$-variation is not given by every sequence of partitions with norms converging to 0 . Goffman and Loughlin noted in [3] that for every continuous $f:[a, b] \rightarrow \mathbf{R}$ and $p>1$ there is a sequence of partitions $a=t_{0}^{k}<t_{1}^{k}<\ldots<t_{n_{k}}^{k}=b$, with norms converging to 0 , for which

$$
\lim _{k \rightarrow \infty} \sum_{i=1}^{n}\left|f\left(t_{i}^{k}\right)-f\left(t_{i-1}^{k}\right)\right|^{p}=0 .
$$

A similar property holds for the general case $W^{(N, d)}$. Let $p>2$ and choose $\delta$ so that $1 / p<\delta<1 / 2$. Choose $q$ such that $q>1$ and $q>(N-1) /(p \delta-1)$. Divide $U^{N}$ into $h$-cubes (cubes with edges of length $h$ ). On each $h$-cube divide one edge into intervals of length $h^{q}$. Hyperplanes through these points divide the $h$-cube into rectangles. There are $b^{1-q}$ of the rectangles in each $h$-cube. By the Lipschitz condition the $N$ th order difference $Y$ defined on each of these "thin" rectangles satisfies $|Y|<2^{N-1} K h^{q \delta}$. Summing over all the rectangles,

$$
\sum|Y|^{p}<\left(2^{N-1} K\right)^{p} h^{q \delta p-N-q+1},
$$

which converges to 0 as $h$ goes to 0 .

3. This fact motivates us to define another kind of variation, which we call weak variation. Our first definition of weak variation is as follows. Let $\pi=\left\{A_{i}\right\}$ be a countable covering of $U^{N}$, the unit $N$-cube. We define the weak $p$-variation of a function $X$ to be

$$
V_{p}^{0}(X)=\lim _{\epsilon \rightarrow 0} \inf _{\|\pi\|<\epsilon} \sum_{i}\left(\delta\left(X_{A_{i}}\right)\right)^{p}
$$

where $\delta\left(X_{A_{i}}\right)$ is the diameter of the image of $A_{i}$, and

$$
\|\pi\|=\sup \left\{\text { diameter of } A_{i}: A_{i} \in \pi\right\} \text {. }
$$

This variation yields a weak variation dimension of $X, \operatorname{dim}_{0}(X)$, in the same way as described for the strong variation.

Theorem 2. For any continuous function $X: U^{N} \rightarrow \mathbf{R}^{d}, \operatorname{dim}_{0}(X)=$ 
$\operatorname{dim}_{H}\left(X_{U N}\right)$, the Hausdorff dimension of the image of $U^{N}$.

Proof. Let $\pi_{k}=\left\{A_{i}^{k}\right\}$ be a sequence of coverings of $U^{N}$ such that $\lim _{k \rightarrow \infty}\left\|\pi_{k}\right\|=0$ and

$$
\lim _{k \rightarrow \infty} \sum_{i}\left(\delta\left(X_{A_{i}^{k}}^{k}\right)\right)^{p}=V_{p}^{0}(X)
$$

But the Hausdorff $p$-measure of $X_{U N}$ is not greater than $\lim _{k \rightarrow \infty} \Sigma_{i}\left(\delta\left(X_{A_{i}^{k}}\right)\right)^{p}$, so $\operatorname{dim}_{0}(X) \geq \operatorname{dim}_{H}\left(X_{U N}\right)$.

To show $\operatorname{dim}_{0}(X) \leq \operatorname{dim}_{H}\left(X_{U N}\right)$ it suffices to show that for each $p$, $H_{p}\left(X_{U N}\right)=0$ implies $\bar{V}_{p}^{0}(X)=0$. ( $H_{p}$ is the Hausdorff $p$-measure.) Let $\epsilon>0$. If $H_{p}\left(X_{U N}\right)=0$, there is a covering $\left\{A_{i}\right\}$ of $X_{U N}$ such that $\Sigma_{i}\left(\delta\left(A_{i}\right)\right)^{p}<\epsilon^{N+1}$. Divide $U^{N}$ into $\epsilon$-cubes. Label the cubes $B_{j}, j=$ $1, \cdots, \epsilon^{-N}$. Let $C_{i j}=X^{-1}\left(A_{i}\right) \cap B_{j}$. Then

$$
V_{p}^{0}(X) \leq \lim _{\epsilon \rightarrow 0} \sum_{i j}\left(\delta\left(X_{C_{i j}}\right)\right)^{p} \leq \lim _{\epsilon \rightarrow 0} \sum_{j=1}^{\epsilon-N} \sum_{i}\left(\delta\left(A_{i}\right)\right)^{p} \leq \lim _{\epsilon \rightarrow 0} \epsilon^{-N} \epsilon^{N+1}=0 .
$$

4. The last part of the above proof required disconnected sets in the coverings. This motivates a second definition of weak variation, in which the sequences of coverings are required to satisfy a parameter of regularity. (A sequence $\left\{\pi_{k}\right\}$ of coverings satisfies a parameter of regularity $K>0$ if each set $A$ in each $\pi_{k}$ satisfies $\sup _{B \subset A} \delta(B) / \delta(A) \geq K$, where $B$ is a ball.) For a sequence $\pi_{k}=\left\{A_{i}^{k}\right\}$ of coverings of $U^{N}$ with $\lim _{k \rightarrow \infty}\left\|\pi_{k}\right\|=0$, let

$$
V\left(p, X,\left\{\pi_{k}\right\}\right)=\lim _{k \rightarrow \infty} \inf \sum_{i}\left(\delta\left(X_{A_{i}^{k}}\right)\right)^{p}
$$

and define the weak $p$-variation of $X$ to be

$$
V_{p}^{w}(X)=\inf \left\{V\left(p, X,\left\{\pi_{k}\right\}\right):\left\{\pi_{k}\right\} \text { satisfies some parameter of regularity }\right\} .
$$

(Different sequences of partitions may have different parameters of regularity.) As before, the weak $p$-variation yields a weak variation dimension of $X, \operatorname{dim}_{w}(X)$.

Theorem 3. If $X=W^{(N, d)}$, then $\operatorname{dim}_{w}(X)=2 N$ with probability one.

Proof. The inequality $\operatorname{dim}_{w}(X) \leq 2 N$ follows from the Lipschitz condition. Let $p>2 N$ and $\delta=N / p$. For any sequence of partitions $\pi_{k}=\left\{A_{i}^{k}\right\}$ of $U^{N}$ satisfying a parameter of regularity and $\lim _{k \rightarrow \infty}\left\|\pi_{k}\right\|=0$,

$$
V_{p}^{w}(X) \leq \liminf _{k \rightarrow \infty} \sum_{i}\left(\delta\left(X_{A_{i}^{k}}\right)\right)^{p} \leq \liminf _{k \rightarrow \infty} \sum_{i}\left(K \delta\left(A_{i}^{k}\right)\right)^{N} .
$$


This sum is bounded since $\left\{\pi_{k}\right\}$ satisfies a parameter of regularity and $U^{N}$ has finite Hausdorff $N$-measure.

To show $\operatorname{dim}_{w}(X) \geq 2 N$ we use the facts that for $X=W^{(N, d)}$,

(a) $\operatorname{dim}_{H}(\operatorname{gr} X)=\min \{2 N, N+d / 2\}$, and

(b) $\operatorname{dim}_{H}\left(X_{U N}\right)=\min \{2 N, d\}$ with probability one. (See appendix.)

Here $\operatorname{dim}_{H}(\operatorname{gr} X)$ denotes the Hausdorff dimension of the graph of $X$. As in Part 3 we have $H_{p}\left(X_{U N}\right) \leq V_{p}^{w}(X)$, so if $2 N \leq d$, (b) implies $\operatorname{dim}_{w}(X) \geq$ $\operatorname{dim}_{H}\left(X_{U N}\right)=2 N$ with probability one. lowing

If $2 N \geq d$, the inequality $\operatorname{dim}_{w}(X) \geq 2 N$ follows from (a) and the fol-

Lemma. If $2 N \geq d$, then for each $p>N / d$ and almost every $\omega$, there is a constant $C=C(p, \omega)$ such that

$$
H_{d+N-N / p}(\operatorname{gr} X(\omega)) \leq C\left[V_{d p}^{w}(X)+1\right]^{1 / p} .
$$

Here $H_{q}(\mathrm{gr} X)$ denotes the Hausdorff q-measure of the graph of $X$.

Proof. Let $p>N / d$ and let. $\pi_{k}=\left\{A_{i}^{k}\right\}$ be a sequence of partitions of $U^{N}$ satisfying some parameter of regularity such that $\lim _{k \rightarrow \infty}\left\|\pi_{k}\right\|=0$ and

$$
\lim _{k \rightarrow \infty} \sum_{i}\left(\delta\left(X_{A_{i}^{k}}\right)\right)^{d p}<V_{d p}^{w}(X)+1 .
$$

Let $\delta_{i}^{k}=\delta\left(X_{A_{i}^{k}}\right)$ and $r_{i}^{k}=$ the integer part of $\delta_{i}^{k} / \delta\left(A_{i}^{k}\right)$. Now the image of $A_{i}^{k}$ can be covered by $\left(r_{i}^{k}+1\right)^{d}$ cubes whose edges have length $\delta\left(A_{i}^{k}\right)$. Likewise the graph of $A_{i}^{k}$ can be covered by the same number of cubes of the same size. The estimate of the Hausdorff $d+N-N / p$ measure of the graph of $A_{i}^{k}$ is

$$
H_{i}^{k}=\left(r_{i}^{k}+1\right)^{d}\left(\delta\left(A_{i}^{k}\right)\right)^{d+N-N / p} .
$$

Since $\left(r_{i}^{k}+1\right)^{d} \leq\left(2 \delta_{i}^{k} / \delta\left(A_{i}^{k}\right)\right)^{d}+1$,

$$
H_{i}^{k} \leq\left(2 \delta_{i}^{k}\right)^{d}\left(\delta\left(A_{i}^{k}\right)\right)^{N-N / p}+\left(\delta\left(A_{i}^{k}\right)\right)^{d+N-N / p} .
$$

Now the kth estimate of the $d+N-N / p$ measure of the graph of $X$ is

$$
\sum_{i} H_{i}^{k} \leq 2^{d} \sum_{i}\left(\delta_{i}^{k}\right)^{d}\left(\delta\left(A_{i}^{k}\right)\right)^{N-N / p}+\left\|\pi_{k}\right\|^{d-N / p} \sum_{i}\left(\delta\left(A_{i}^{k}\right)\right)^{N} .
$$

By Hölder's inequality,

$$
\sum_{i}\left(\delta_{i}^{k}\right)^{d}\left(\delta\left(A_{i}^{k}\right)\right)^{N-N / p}<\left[\sum_{i}\left(\delta_{i}^{k}\right)^{d p}\right]^{1 / p}\left[\sum_{i}\left(\delta\left(A_{i}^{k}\right)\right)^{N}\right]^{1-1 / p} .
$$


The sum $\Sigma_{i}\left(\delta\left(A_{i}^{k}\right)\right)^{N}$ is bounded (say by $M$ ) for all $k$ since the sequence $\left\{\pi_{k}\right\}$ satisfies a parameter of regularity and the Hausdorff $N$-measure of $U^{N}$ is finite. So

$$
\begin{aligned}
H_{d+N-N / p}(\operatorname{gr} X) & \leq \limsup _{k \rightarrow \infty} \sum_{i} H_{i}^{k} \\
& \leq \limsup _{k \rightarrow \infty} 2^{d} M^{1-1 / p}\left[\sum_{i}\left(\delta_{i}^{k}\right)^{d p}\right]^{1 / p}+M \lim _{k \rightarrow \infty}\left\|\pi_{k}\right\|^{d-N / p} \\
& \leq 2^{d} M^{1-1 / p}\left[V_{d p}^{w}(X)+1\right]^{1 / p} .
\end{aligned}
$$

I would like to thank Professor Casper Goffman for suggesting this problem.

Appendix. The following theorem generalizes results of S. J. Taylor [6], [7], P. Lévy [4], and A. S. Besicovitch and H. D. Ursell [2], and the proof is summarized here in three parts.

Theorem. The Hausdorff dimensions of the graph and image of $W^{(N, d)}$ are almost surely $\min \{2 N, N+d / 2\}$ and $\min \{2 N, d\}$, respectively.

I. Let $X: U^{N} \rightarrow \mathbf{R}^{d}$ belong to the Lipschitz $\delta$-class (Lip $\delta$ ). Then $\operatorname{dim}_{H}\left(X_{U}\right) \leq \operatorname{dim}_{H}(\operatorname{gr} X) \leq \min \{N / \delta, N+(1-\delta) d\}$. (Since $W^{(N, d)}$ is almost surely in Lip $\delta$ for every $\delta<1 / 2[5]$, we obtain part of the theorem.)

For the proof of I, divide $U^{N}$ into $h$-cubes (cubes with edge of length $b)$. The graph of each $h$-cube is contained in a set of diameter $K_{1} h^{\delta}$. The estimate of the $N / \delta$ measure of the graph is $b^{-N}\left(K_{1} b^{\delta}\right)^{N / \delta}=K_{2}<\infty$. On the other hand, dividing the image of each $h$-cube into $h$-cubes provides a covering of the graph with $h$-cubes, and the estimate of the $N+(1-\delta) d$ measure of the graph is

$$
h^{-N}\left[K_{1} h^{\delta-1}\right]^{d}\left[(N+d)^{1 / 2} h\right]^{N+(1-\delta) d}=K_{2}<\infty .
$$

II. Let $X=W^{(N, d)}, 2 N \leq d$. Then $\operatorname{dim}_{H}($ gr $X) \geq \operatorname{dim}_{H}\left(X_{U N}\right) \geq 2 N$. Let $a<2 N$. It is sufficient to show that the a capacity of the image of $U^{N}$, $C_{a}\left(X_{U N}\right)$, is positive. For this it is sufficient to show

$$
\int_{U} N \int_{U} N \frac{d s d t}{|X(s, \omega)-X(t, \omega)|^{a}}<\infty .
$$

Now 


$$
\begin{aligned}
\int_{\mathbf{\Omega}} \frac{d \omega}{|X(t, \omega)|^{\alpha}} & =(2 \pi|t|)^{-d / 2} \int_{\mathbf{R}^{d}}|u|^{-\alpha} \exp -\left(\frac{u_{1}^{2}+\cdots+u_{d}^{2}}{2|t|}\right) d u \\
& =K_{1}|t|^{-d / 2} \int_{0}^{\infty} r^{d-1-\alpha} \exp \left(\frac{-r^{2}}{2|t|}\right) d r \\
& =K_{1}|t|^{-\alpha / 2} \int_{0}^{\infty} x^{d-1-\alpha} \exp \left(\frac{-x^{2}}{2}\right) d x,
\end{aligned}
$$

where $r=|t|^{1 / 2} x$. The integral is finite since $a<d$, so

$$
\int_{U} N \int_{U^{N}} d s d t \int_{\Omega} \frac{d \omega}{|X(s, \omega)-X(t, \omega)|^{a}}=\int_{U^{N}} \int_{U^{N}} K \frac{d s d t}{|s-t|^{a / 2}}
$$

which is finite since $\alpha / 2<N$. Fubini's theorem gives the desired conclusion. A similar argument shows $\operatorname{dim}_{H}\left(X_{U N}\right) \geq d$ whenever $2 N \geq d$.

III. If $2 N>d$, then $\operatorname{dim}_{H}(\mathrm{gr} X) \geq N+d / 2$. Let $a$ be any number satisfying $d \leq \alpha<N+d / 2$, and choose $\epsilon>0$ so that $\alpha+\epsilon<d+N / d$. We show $C_{\alpha}(g r X)>0$. Let $r(t, \omega)=\left[|X(t, \omega)|^{2}+|t|^{2}\right]^{1 / 2}$ and $f(t, R)=$ $P\{\omega: r(t, \omega)<R\}$. If $R \geq|t|>0$,

$$
f(t, R)=K_{1}|t|^{-d / 2} \int_{0}^{\left(R^{2}-|t|^{2}\right)^{1 / 2}} r^{d-1} \exp \left(\frac{-r^{2}}{2|t|}\right) d r
$$

If $|t| \geq R>0, f(t, R)=0$. We have

$$
\begin{aligned}
\int_{\Omega} \frac{d \omega}{|r(t, \omega)|^{a}} & =\int_{0}^{\infty} R^{-a} \frac{\partial f}{\partial R} d R \\
& =K_{2}|t|^{-d / 2} \int_{|t|}^{\infty} R^{1-a}\left(R^{2}-|t|^{2}\right)^{d / 2-1} \exp \left(-\frac{R^{2}-|t|^{2}}{2|t|}\right) d R .
\end{aligned}
$$

The substitution $R^{2}=|t| x^{2}+|t|^{2}$ gives

$$
\int_{\Omega} \frac{d \omega}{|r(t, \omega)|^{\alpha}} \leq K_{3}|t|^{d / 2-\alpha-\epsilon} \int_{0}^{\infty} x^{2 \epsilon-1} \exp \left(-\frac{x^{2}}{2}\right) d x=K|t|^{d / 2-a-\epsilon} .
$$

Now

$\int_{U^{N}} \int_{U^{N}} d s d t \int_{\Omega} \frac{d \omega}{|(s, X(s, \omega))-(t, X(t, \omega))|^{a}} \leq \int_{U^{N}} \int_{U^{N}} \frac{K d s d t}{|s-t|^{a+\epsilon-d / 2}}$.

This integral is finite since $a+\epsilon-d / 2<N$, and Fubini's theorem again concludes the proof. 


\section{REFERENCES}

1. Simeon M. Berman, A version of the Lévy-Baxter theorem for the increments of Brownian motion of several parameters, Proc. Amer. Math. Soc. 18 (1967), 1051-1055.

2. A. S. Besicovitch and H. D. Ursell, Sets of fractional dimension. V: $O_{n}$ dimensional members of some continuous curves, J. London Math. Soc. 12 (1937), $18-25$.

3. C. Goffman and J. Loughlin, Strong and weak $\Phi$-variation of Brownian motion, Indiana Univ. Math. J. 22 (1972/73), 135-138. MR 45 \#5288.

4. P. Lévy, La mesure de Hausdorff de la courbe du mouvement Brownien, Giorn. Ist. Ital. Atturi 16 (1953), 1-37. MR 16, 268.

5. - Processus stochastiques et mouvement Brownien, Gauthier-Villars, Paris, 1948, p. 265. MR 10, 551.

6. S. J. Taylor, The Hausdorff $\alpha$-dimensional measure of Brownian paths in n-space Proc. Cambridge Philos. Soc. 49 (1953), 31-39. MR 14, 663.

7. — The a-dimensional measure of the graph and set of zeros of a Brownian path, Proc. Cambridge Philos. Soc. 51 (1955), 265-274. MR 17, 595. 47907

DEP ARTMENT OF MATHEMATICS, PURDUE UNIVERSITY, LAF AYETTE, INDIANA

Current address: Department of Mathematics, University of Hawaii, Honolulu, Hawaii 96822 